\title{
Microchanneled Chirped Fibre Bragg Gratings For Simultaneous Refractive Index and Temperature Measurements
}

\author{
Pouneh Saffari ${ }^{1}$, Hongyan $\mathrm{Fu}^{2}$, Kaiming $\mathrm{Zhou}^{1}$, Lin Zhang ${ }^{1}$ and Ian Bennion ${ }^{1}$ \\ ${ }^{1}$ Photonic Research Group, Aston University, Birmingham, B4 7ET, United Kingdom \\ ${ }^{2}$ Center for Optical and Electromagnetic Research, Zhejiang University, China \\ Email: saffarip@aston.ac.uk
}

\begin{abstract}
We report here the fabrication, charaterisation and refractive index sensing of two microchanneled chirped fiber Bragg gratings (MCFBGs) with different channel sizes $(\sim 550 \mu \mathrm{m}$ and $\sim 1000 \mu \mathrm{m})$. The chirped grating structures were UVinscribed in optical fibre and the microchannels were created in the middle of the CFBGs by femtosecond $\left(f_{s}\right)$ laser assisted chemical etching method. The creation of microchannels in the CFBG structures gives an access to the external index liquid, thus inducing refractive index (RI) sensitivity to the structure. In comparison with previously reported FBG based RI sensors, for which the cladding layers usually were removed, the MCFBGs represent a more ideal solution for robust devices as the microchannel will not degrade the structure strength. The two MCFBGs were spectrally charaterised for their RI and temperature responses and both gratings exhibited unique thermal and RI sensitivities, which may be utilised for implementation of bio-chemical sensors with capability to eliminate temperature crosssensitivity.
\end{abstract}

Keywords: Chirped fibre Bragg gratings (CFBGs), microchannel, femtosecond laser, optical fibre sensor, simultaneous refractive index and temperature sensing

\section{INTRODUCTION}

Today, advanced sensing devices and systems capable of multi-parameter sensing operation are required due to the necessity of monitoring physical, chemical, and biological properties in natural environments, industrial processes and many other applications ${ }^{1}$. For this demand, fibre-optic sensors can offer unique solutions over conventional technologies. Over the last two decades, fibre Bragg grating (FBG) based sensors have attracted considerable attention, owing to their enormous advantageous over traditional optical sensors, such as compactness, low cost fabrication, multiparameter measurement capability, ease of interrogation, high sensitivity, remote sensing and so on. FBG sensors have been used for measurement of a wide range of physical parameters, including strain, temperature, pressure, bending and many others. However, since the mode coupling takes place between the well bounded counter-propagating core modes, normal FBGs are intrinsically insensitive to the surrounding medium and consequently are not applicable directly as refractive index (RI) sensors. In order to measure surrounding medium refractive index, it is important for the mode field to penetrate evanescently into the surrounding medium. Modified FBG structures have been proposed to implement RI sensors. For example, thinning the FBG cladding layers by chemical etching have been reported to induce RI sensitivity to the structures ${ }^{2-7}$. In most demonstrated structures, the cladding of the fibre is almost completely removed through the process of chemical etching, resulting in severe degradation of the device strength.

In recent years, the technique of laser inscription in transparent dielectric materials by means of tightly focused femtosecond $(f s)$ laser irradiation has attracted much research attention and been deemed as one of the most efficient approaches to inscribing microstructures onto silica materials/fibres, due to its high peak power and spatial resolution. $f_{s}$ laser has been adopted to fabricate microchannels on the optical fiber ${ }^{8}$. It has been reported that regions modified by $f_{s}$ laser have much higher etching rate than the pristine material, of which the contrast ratio can be up to $100: 1^{9}$. Also, a recent study has reported the fabrication of a micro-slot superimposed on an FBG utilising the same technique ${ }^{10}$. The $f S^{-}$

Optical Sensors 2009, edited by Francesco Baldini, Jiri Homola, Robert A. Lieberman, Proc. of SPIE Vol. 7356, $735604 \cdot$ ㄷ 2009 SPIE · CCC code: 0277-786X/09/\$18 · doi: 10.1117/12.820651 
inscription/etching combined approach represents a low-cost but effective microstructuring technique compared to $f_{s}$ laser direct machining. In this paper, we report the fabrication of two microchanneled chirped fiber Bragg gratings (MCFBGs) of different channel sizes $(\sim 550 \mu \mathrm{m}$ and $\sim 1000 \mu \mathrm{m})$ created by using $f_{s}$ laser assisted chemical etching technique. We have investigated their spectral characteristics to surrounding medium refractive index and the temperature changes. The proposed MCFBGs show not only the solution for non strength degrading RI sensors but also enhanced RI sensitivity and unique function to eliminate temperature cross-sensitivity. The proposed MCFBG structures may represent more ideal device candidates for implementation of in-fibre bio-chemical sensors for a range of potential applications.

\section{STRUCTURE DESCRIPTION AND EXPERIMENTAL FABRICATION}

Fig. 1 shows the geometry of the proposed MCFBG, which consists of a chirped fibre Bragg grating (CFBG) in the core and a microchannel created in the middle of the CFBG across the fibre. The length, pitch and the chirp rate of the CFBG are $L_{g}, A$, and $C$ respectively, and $L_{m}$ is the length and $h$ is height of the microchannel structure.

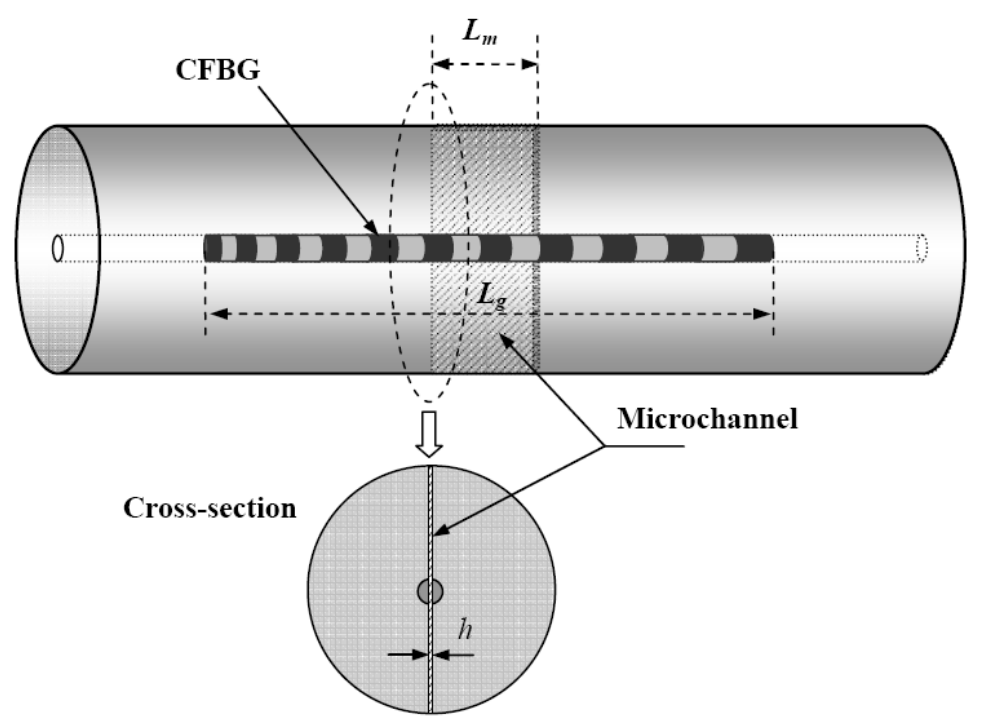

Figure 1. Schematic diagram of the proposed MCFBG consisting of a CFBG in the fibre core, and a microchannel (with the length of $\mathrm{Lm}$ ) created in the middle region of the CFBG across the fibre.

The fabrication process of the proposed MCFBG structure includes three steps:

i. Fabricating CFBGs into the core of a single-mode fibre by UV laser inscription and phase mask scanning technique;

ii. Patterning the designed microchannel in the middle of the CFBGs across the fibre using tightly focused $f_{s}$ laser beam;

iii. Etching the $f_{s}$-modified CFBGs in a 5\% hydrofluoric acid (HF) solution for selective removal of the $f_{s}$-modified region. 
The CFBGs were UV-inscribed in single mode standard telecom fibre (Corning SMF-28) using the scanning phase mask technique with a CW frequency doubled Argon ion laser operating at $244 \mathrm{~nm}$. Prior to the UV-inscription, the photosensitivity of SMF-28 fibre samples were enhanced by loading them in hydrogen tube for a period of 48 hours at a pressure of 150 bars and the temperature at $80^{\circ} \mathrm{C}$. The phase mask used in the experiment is uniformly chirped and has the period and the chirp rate of $1070 \mathrm{~nm}$ and $1.12 \mathrm{~nm} / \mathrm{cm}$. The mask is in a circular shape with the radius of $25 \mathrm{~mm}$. A few $\mathrm{cm}$ of the middle parts of a $60 \mathrm{~mm}$ long host fibre samples were stripped off for grating inscription. The UV beam was scanned across the stripped fibre for $10 \mathrm{~cm}$ length. A UV power of $100 \mathrm{~mW}$ and a scanning speed of $0.025 \mathrm{~mm} / \mathrm{s}$ were used to inscribe CFBGs in the host fibres. Before subjecting the CFBG fibres for $f s$ laser patterning, we measured the CFBGs' spectra using a broadband source and an optical spectrum analyser and the measured central Bragg wavelengths of the fabricated CFBGs are around 1550nm with 10nm bandwidth.

For microchannel patterning process, the $f s$ laser pulses of $800 \mathrm{~nm}$ were tightly focused on the fibre using a $\times 100$ objective lens (with an N.A. of 0.55 and a working distance of $13 \mathrm{~mm}$ ). The pulse width of $f s$ laser was measured to be about 150fs, and the repetition rate was at $1 \mathrm{kHz}$. The fibre containing CFBG was mounted on a 3-D axis air-bearing translation stage, and the microchannel profile was written by moving the stage along the cross-section of the fibre around the middle of the CFBG region. Using this method, two microchannels of sizes $550 \times 2 \times 125 \mu \mathrm{m}^{3}$ and $1000 \times 2 \times 125 \mu \mathrm{m}^{3}$ were written on two CFBGs. Following the $f_{s}$-inscription, the MCFBG patterned with $550 \mu \mathrm{m}$ channel was chemically etched in the HF solution for only $\sim 8 \mathrm{~min}$ while the MCFBG with $1000 \mu \mathrm{m}$ was etched for $15 \mathrm{~min}$ to create the designed microchannels. An ultrasonic bath was employed to enhance the penetration of HF solution into the $f_{s}$ laser pre-treated area during the etching.

After the chemical etching process, a high-resolution optical microscope was used to inspect the MCFBG structures. Fig. 2(a) shows the examined 550 $\mu \mathrm{m}$ microchannel image. From Fig. 2(a) we can see that the measured microchannel length is $557.03 \mu \mathrm{m}$, which is in good agreement with the design size $550 \mu \mathrm{m}$. The transmission spectrum of this $550 \mu \mathrm{m}-$ MCFBG is shown in Fig. 2(b), giving a typical phase shift feature, as we see there are a main transmission peak and several interferences on the shorter wavelength side of the main peak. This resulted MCFBG spectrum may be explained by the combining effect from three sub gratings - two $\sim 5 \mathrm{~mm}$ chirped gratings separated by a very short $550 \mu \mathrm{m}$ grating with a microchannel. The microchannel will reduce the refractive index of the short grating, thus shift its reflection band to the shorter wavelength side slightly, resulting in the generation of the main transmission peak in the original spectral part. The shifted reflection of the short grating partially overlaps with the sub-CFBG on the shorter wavelength side, thus generating strong interferences (sub transmission peaks) in the overlap spectral region. 

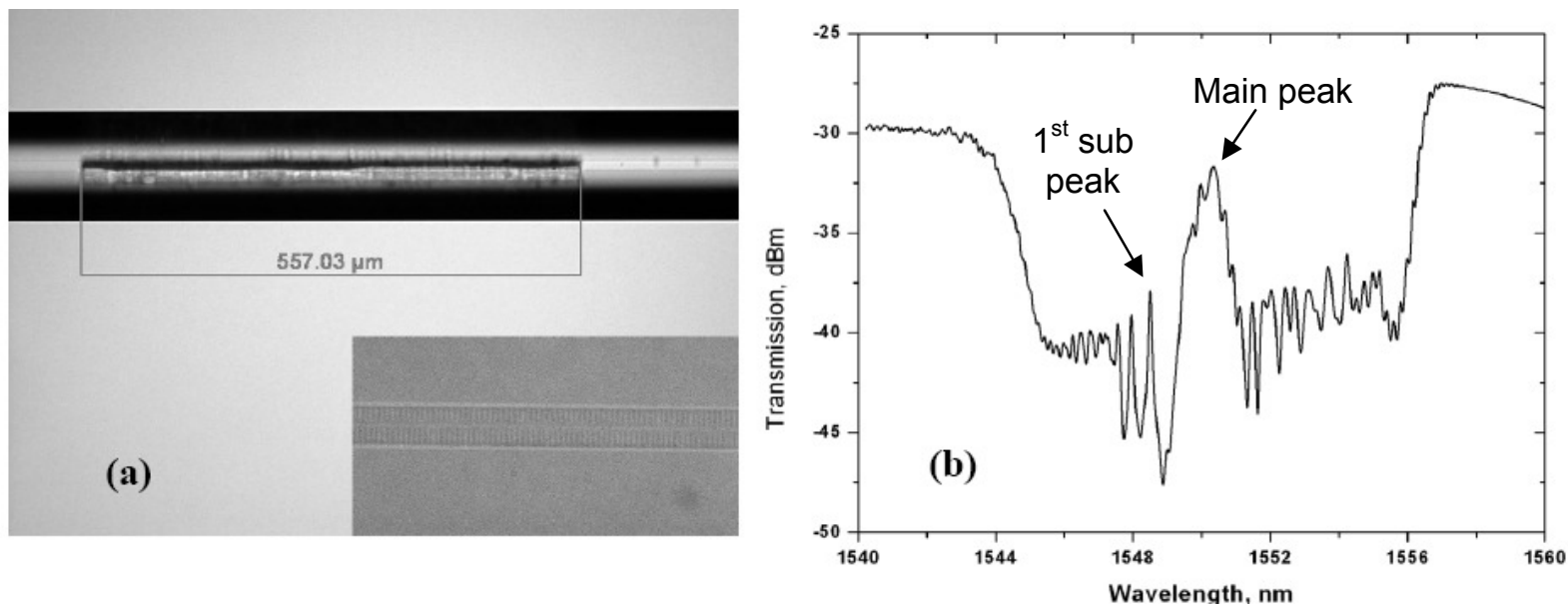

Figure 2. (a) Microscope image of the $550 \mu \mathrm{m}-\mathrm{MCFBG}$ under a $\times 10$ microscopic lens; Inset: image of the chirped grating structure. (b) Transmission spectrum of the MCFBG (I: main transmission peak; II: 1st interference peak).

Fig. 3(a) and (b) show the entire and zoomed transmission spectrum of the $1000 \mu \mathrm{m}-\mathrm{MCFBG}$, respectively. From the figures we can see that a slightly narrower main transmission peak is created in the centre of the stopband of the original CFBG and more fine interference features are present in the stopband of the CFBG. This MCFBG structure can also be regarded by the formation of three sub gratings, but the middle short grating now is twice long of that in $550 \mu \mathrm{m}-$ MCFBG. The longer short grating will have a narrower reflection spectrum, which may shift more to the shorter wavelength side, leaving absence of grating spectrum in the middle and interferences in the stopband.
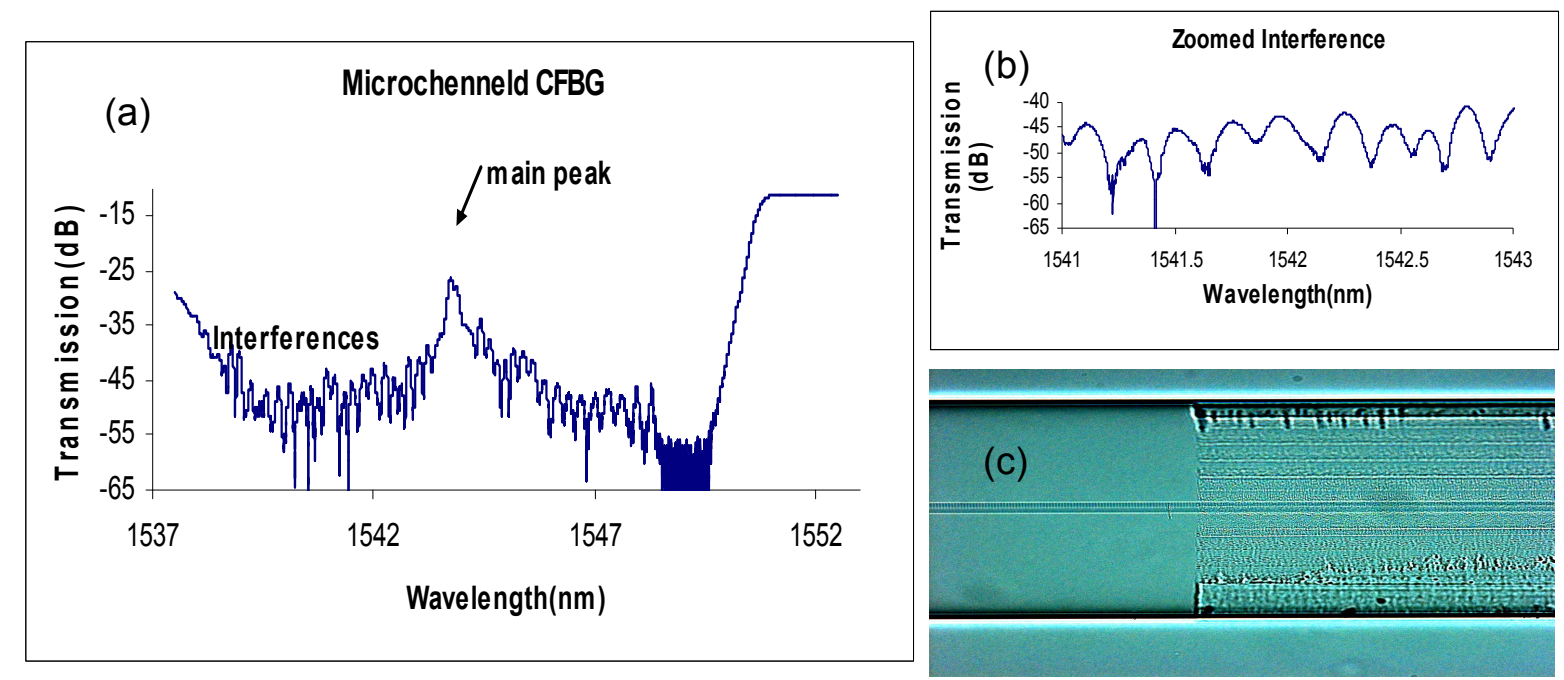

Figure 3. (a) Transmission spectrum of the $1000 \mu \mathrm{m}-\mathrm{MCFBG}$; (b) Zoomed interferences in the stopband. (c) Microscope image of the edge of the microchannel in the $1000 \mu \mathrm{m}-\mathrm{MCFBG}$ under a $\times 401000 \mu \mathrm{m}$ oil-immersion microscopic lens. 
The creation of the microchannels in the CFBGs is to enable the grating in the core to be penetrated by the surrounding medium, thus having RI responsivity for bio-chemical sensing. The small size of the microchannel

will not just induce the access to liquid solution but also maintain the robustness of the device, which is much more desirable compared to those FBG devices with cladding layers fully removed by etching treatment.

\section{SURRONDING MEDIUM REFRACTIVE INDEX AND TEMPERATURE MEASUREMENT}

The creation of a microchannel in the CFBG structure results in the effective index change due to the infusion of the surrounding medium in this region ${ }^{10}$. As shown in Fig. 2(b) and 3(b), the microchannels in the two CFBGs have generated a main and sub transmission peaks in the reflection bands of the original CFBGs. One can consider that in the area of the grating where the microchannel has been created, the reduced refractive index of this short grating at that part will shift its reflection band to the shorter wavelength side, resulting in the generation of the main and sub transmission peaks in the original reflection band of the CFBG. If liquid with different RIs are filled in the microchannel the spectrum of the MCFBG will change accordingly, thus induce RI sensitivity.

We have characterised the two MCFBGs for their RI response properties. In the experiment, the MCFBG fibre was fixed in a V-grooved aluminum plate, which ensured the measurement free from other effects, such as strain, bend and temperature. A series of index liquids (from Cargille Laboratories) were used as surrounding media for investigation. Each index liquid was pulled into the groove to immerse the MCFBG and the corresponding spectrum was measured by a broadband source and an optical spectrum analyser. After each measurement, the MCFBG was rinsed with acetone, methanol and water for several times until the original spectrum was restored, making sure no residue index liquid left in the microchannel region and ready for next index liquid to be applied.

\subsection{0 $\mu \mathrm{m}-\mathrm{MCFBG}$}

In the case of the $550 \mu \mathrm{m}-\mathrm{MCFBG}$, due to reduced effective index, the reflection spectrum of the microchanneled short grating in the middle will blue-shift to overlap with the spectrum of the sub-CFBG on the shorter wavelength side. Because the middle grating is very short, its spectrum is relatively broad, thus only partially overlap with the shorter wavelength side sub-CFBG. As a result, the main and sub transmission peaks generated by this structure should both sensitive to RI change. To evaluate the RI response, we measure the wavelength shifts of both main and the 1st sub transmission peak against the RI changes and the results are plotted in Fig. 4.

As expected, both main and sub transmission peaks shifted with RI with a non-linear characteristic behaviour. For the main transmission peak, the RI sensitivity is about $1 \mathrm{~nm} / \mathrm{RIU}$ in the low RI region from 1.34 to 1.42 and $10 \mathrm{~nm} / \mathrm{RIU}$ in the high RI region from 1.42 to 1.46 , whereas for the 1 st sub peak, is $0.1 \mathrm{~nm} / \mathrm{RIU}$ and $1 \mathrm{~nm} / \mathrm{RIU}$, respectively. This indicates that the RI change induces more significant shift to the main peak than to the sub one. 


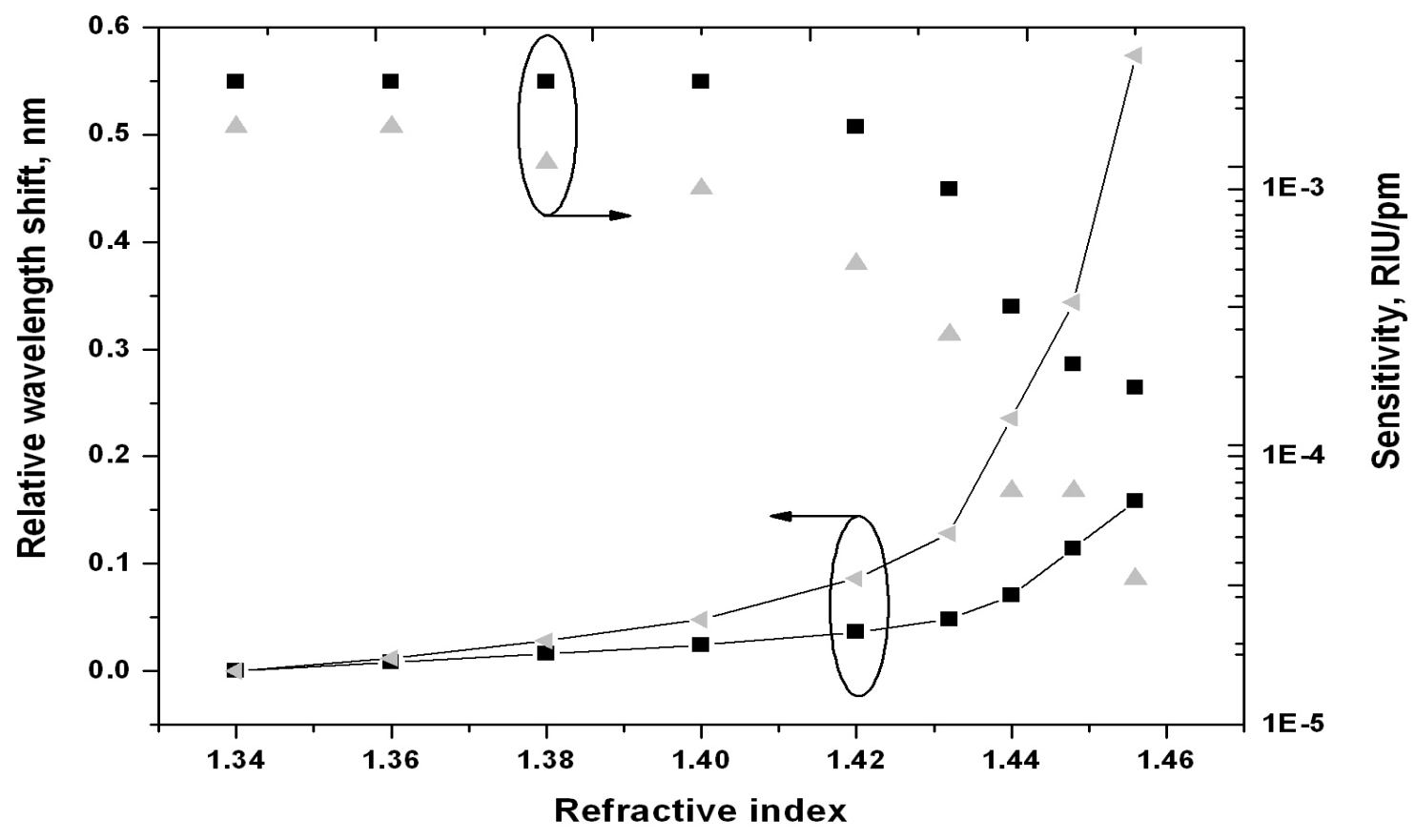

Figure 4. The wavelength shift and sensitivity of the main transmission peak (grey triangles) and the first sub transmission peak (black rectangles) against surrounding medium refractive index.

The $550 \mu \mathrm{m}-\mathrm{MCFBG}$ spectral response to temperature was also characterised in the experiment. The MCFBG was housed on a heating base with a temperature controller (Lightwave LTD 5910B). The measured wavelength shift against the temperature is shown in Fig. 5. When the MCFBG is surrounded by air (Fig.5a), the measured wavelength shifts against temperature for the main and 1st sub transmission peaks are similar, giving a temperature sensitivity of $\sim 10 \mathrm{pm} /{ }^{\circ} \mathrm{C}$. In this case, the temperature response is mainly determined by the thermo-optic coefficient of the fibre, and the whole fibre experiences a similar temperature effect. When the MCFBG is immersed in the index liquid (Fig. 5(b), the RI of the liquid is 1.448), both peaks exhibit reduced thermal responsivity due to the negative thermo-optic coefficient of the index liquid. More noticeably, the index liquid in the microchannel affects the main and sub transmission peaks quite differently, as we see that the thermal response $\left(\sim 4 \mathrm{pm} /{ }^{\circ} \mathrm{C}\right)$ of the former is only a half of that $\left(\sim 8 \mathrm{pm} /{ }^{\circ} \mathrm{C}\right)$ of the latter. In combining with their RI sensitivities, this distinct different thermal response of the main and sub transmission peaks could provide effective simultaneous measurement of RI and temperature using such an MCFBG. 

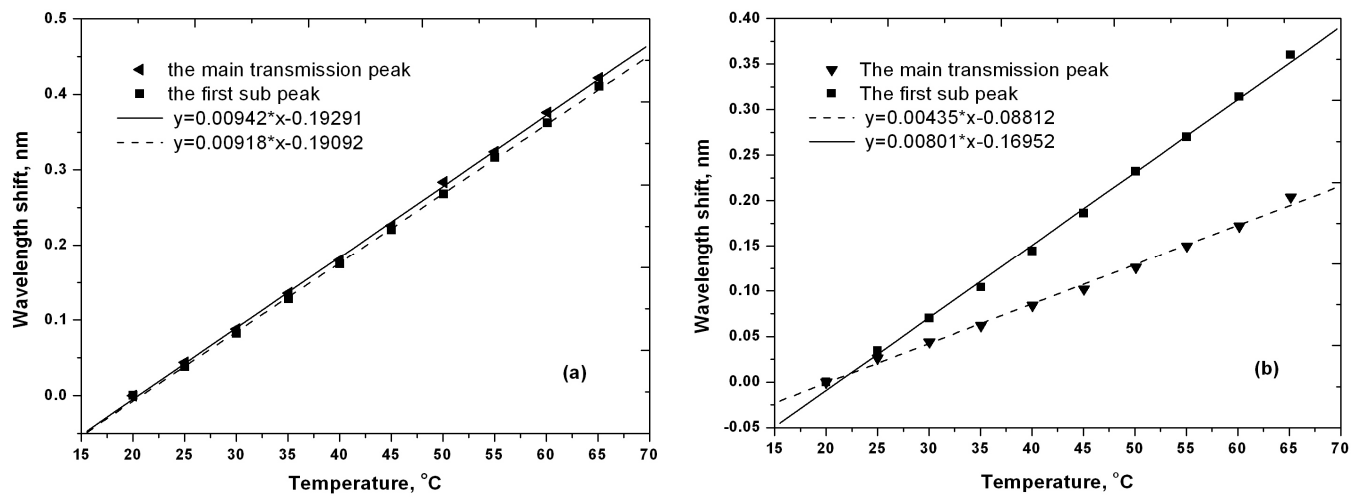

Figure 5. The measured wavelength shift against temperature without external index liquid (a) and with the index liquid of $\mathrm{RI}=1.448$ (b).

\section{$3.21000 \mu \mathrm{m}-\mathrm{MCFBG}$}

For RI characterisation of the $1000 \mu \mathrm{m}-\mathrm{MCFBG}$, we applied different index gels to the structure and measured the wavelength shifts of the main and one of the interference peaks on the shorter wavelength region and saw a quite

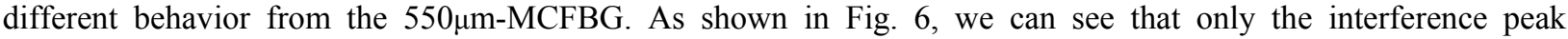
responses to RI change and the main transmission peak in the middle of the stopband is almost insensitive to RI. This is in contrast to the $550 \mu \mathrm{m}-\mathrm{MCFBG}$, as there both main and sub transmission peaks are sensitive to RI. This difference may be explained by the fact that the middle short grating in the $1000 \mu \mathrm{m}-\mathrm{MCFBG}$ is twice long of that in the $500 \mu \mathrm{m}-\mathrm{MCFBG}$, thus resulting in a narrower reflection peak whose reflection spectrum overlaps with the subCFBG spectrum on the shorter wavelength side. As explained before, this will result in absence of grating spectrum in the middle of the stopband of the original CFBG, forming the apparent main transmission peak in the middle of the spectrum. Thus, this apparent main transmission peak will not be affected by the index liquid in the microchannel, i.e. being insensitive to RI. As shown in Fig.6, the RI sensitivity response of the measured interference is also nonlinear, giving an average sensitivity of $1.32 \mathrm{~nm} / \mathrm{RIU}$ in the low RI region from 1.34 to 1.39 and $3.29 \mathrm{~nm} / \mathrm{RIU}$ in the high RI region from 1.39 to 1.44 . These two values are much higher than that of the $1^{\text {st }}$ sub transmission peak in the $550 \mu \mathrm{m}-\mathrm{MCFBG}$.

In comparison with the $550 \mu \mathrm{m}-\mathrm{MCFBG}$, this MCFBG with slightly broad microchannel appears to have more advantages as an RI sensor, as only its interference peaks are sensitive to RI change and the main peak is totally insensitive to RI. Thus, in this structure, the main peak can offer a temperature reference function and using this function, the temperature cross-sensitivity on RI measurement from the interference peak can be eliminated. 


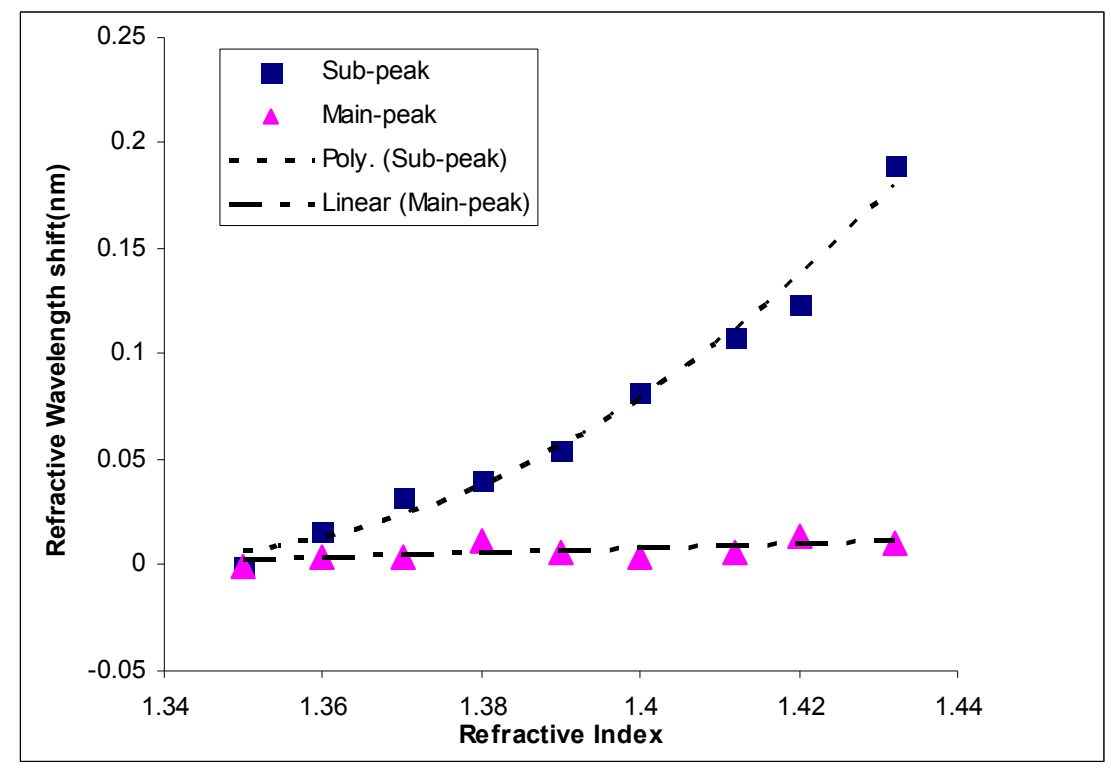

Figure.6. Wavelength shift of the main transmission peak (triangles) and the measured sub interference peak (squares) against surrounding medium refractive index change, showing only the latter is sensitive to the RI change.

\section{CONCLUSIONS}

In this paper, two MCFBGs of different channel sizes, $500 \mu \mathrm{m}$ and $1000 \mu \mathrm{m}$, have been proposed and fabricated by using the $f s$-laser aided chemical etching technique. The reduced effective index of the microchannel region resulting in transmission bands inside the reflection band of the MCFBGs is sensitive to SRI variation. Normal structure FBGs and CFBGs are intrinsically insensitive to external RI change, as the mode coupling occurs between well bounded core modes and has no interaction with surrounding medium. Hence, it is not common to use FBGs and CFBGs for refractive index measurements. Although, some attempts have been reported to remove the cladding layer of FBGs to make them sensitive to surrounding medium RI, this un-doubt will degrade the strength of the device and not be suitable for real applications. In our proposed MCFBG structure, very small size microchannels are created in the gratings to introduce access to the sensing solution, which will not degrade the whole structure, thus maintaining the device strength. Our characterisation results show that the MCFBGs of different microchannel sizes may give different thermal and RI

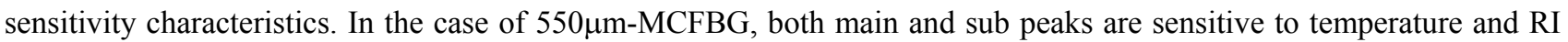
but with distinct different sensitivities. The different sensitivities may be utilised for simultaneous measurement of temperature and RI. In the $1000 \mu \mathrm{m}-\mathrm{MCFBG}$ case, the main peak is insensitive to RI, but the interferences are much more sensitive to RI. This may present an even more suitable structure for bio-chemical sensor, since the temperature cross-sensitivity can be easily eliminated by using the main peak spectral information for temperature compensation. We anticipate that further study and development using advanced $f s$-laser inscription and microstructuring technique may result in novel sensor structures with tailored sensing functionalities for applications. 


\section{REFERENCES}

[1] Zhang, L., Zhang, W. and Bennion, I., [In Fibre Optic Sensors], ed. F T S Yu \& S Yin, CRC Press, $1^{\text {st }}$ edition 2002, $2^{\text {nd }}$ edition 2008, ISBN 978-1-4200-5365-4(2002\&2008).

[2] Iadicicco, A., Cusano, A., Cutolo, Bernini, R. and Giordano, M., "Thinned fiber Bragg gratings as high sensitivity refractive index sensors," IEEE Photon. Technol. Lett., vol. 16, no. 4, pp. 1149-1151, (2004).

[3] Iadicicco, A., Campopiano, S., Cutolo, Antonello., Giordano, M. and Cusano, A., "Refractive index sensor based on microstructured fiber Bragg grating" IEEE Photon. Technol. Lett., vol. 17, no. 6, pp. 1250-1252, (2005).

[4] Zhou, K., Chen, X., Zhang, L. and Bennion, I., "Implementation of optical chemsensors based on HF- etched fibre Bragg grating structures”, Meas. Sci. Technol., 17,, 1140-1145, Apr. (2006).

[5] Chen, X., Zhou, K., Zhang, L. and Bennion, I., "Optical chemsensor based on etched tiled Bragg grating structures in multimode fiber”, IEEE Photon. Technol. Lett., 17, 864-866, Apr. (2005).

[6] Zhou, K., Chen, X., Zhang, L. and Bennion, I., "High sensitivity point-probe optical fiber chem-sensors based on cladding etched fiber Bragg gratings", Photonics Europe (SPIE International Conference), France, Apr. 2004, Proc. SPIE Int. Soc. Opt. Eng. 5459, 409-411, Sep.( 2004).

[7] Iadicicco, A., Campopiano, S., Cutolo, Antonello., Giordano, M. and Cusano, A., "Self temperature referenced refractive index (2006).

[8] Lai, Y., Zhou, K., Zhang, L. and Bennion, I., "Mirochannels in conventional single-mode fibers", Opt. Lett., vol. 31, no. 17 , pp. $2559-2561,(2006)$.

[9] Hnatovsky, C., Taylor, R. S., Simova, E., Bhardwaj, V. R., Rayner, D. M. and Corkum, P. B., "Polarization-selective etching in femtosecond laser-assisted microfluidic channel fabrication in fused silica", Opt. Lett., vol. 30, no. 14, pp. 1867-1869, (2005).

[10] Zhou, K., Lai, Y., Chen, X., Sugden, K., Zhang, L. and Bennion, I., "A refractometer based on a micro-slot in a fiber Bragg grating formed by chemically assisted femtosecond laser processing" Opt. Express, vol. 15, no.24,pp.15848-15853,(2007). 